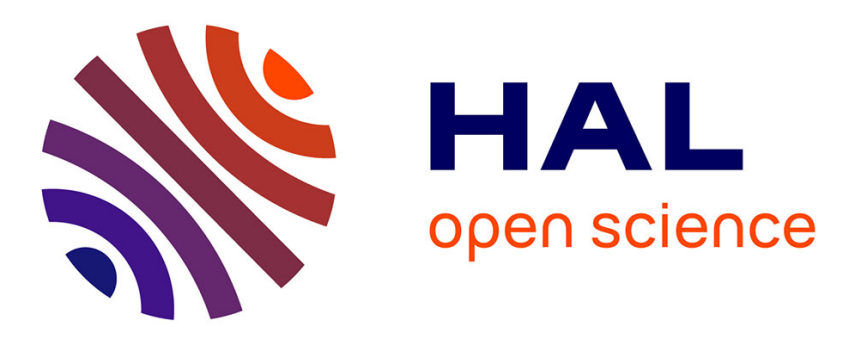

\title{
Distributed DBSCAN Protocol for Energy Saving in IoT Networks
}

\author{
Mazin Kadhum Hameed, Ali Kadhum Idrees
}

\section{To cite this version:}

Mazin Kadhum Hameed, Ali Kadhum Idrees. Distributed DBSCAN Protocol for Energy Saving in IoT Networks. International Conference on Communication, Computing and Electronics Systems, 733, Springer Singapore, pp.11-24, 2021, Lecture Notes in Electrical Engineering, 10.1007/978-98133-4909-4_2. hal-03399772

\section{HAL Id: hal-03399772 \\ https://hal.science/hal-03399772}

Submitted on 24 Oct 2021

HAL is a multi-disciplinary open access archive for the deposit and dissemination of scientific research documents, whether they are published or not. The documents may come from teaching and research institutions in France or abroad, or from public or private research centers.
L'archive ouverte pluridisciplinaire HAL, est destinée au dépôt et à la diffusion de documents scientifiques de niveau recherche, publiés ou non, émanant des établissements d'enseignement et de recherche français ou étrangers, des laboratoires publics ou privés. 


\title{
Distributed DBSCAN Protocol for Energy Saving in IoT Networks
}

\author{
Mazin Kadhum Hameed ${ }^{1}$ and Ali Kadhum Idrees ${ }^{2,}$ \\ Department of Software, University of Babylon, Babylon, Iraq. \\ 1it . mazen.kadhum@uobabylon.edu.iq \\ Department of Computer Science, University of Babylon, Babylon, Iraq \\ 2,*ali.idrees@uobabylon.edu.iq \\ * Corresponding Author
}

\begin{abstract}
Sensor Networks form a crucial topic in research, as it seems to target a huge variety of uses in which it could be applied, such as healthcare, smart cities, environment monitoring, military, industrial automation, and smart grids. The clustering algorithms represent an essential factor in conserving power within energy-constrained networks. The selection of a cluster head balances the energy load within the network in a proper way, eventually contributing to the reduction of energy consumed, as well as the enhancement of network lifespan. This paper introduced a distributed DBSCAN protocol for saving the energy of Sensor devices in IoT Networks. This protocol is implemented on each IoT sensor device and the devices apply the DBSCAN algorithm to partition the network into clusters in a distributed way. The efficient periodic cluster head strategy is proposed based on certain criteria like remaining energy, number of neighbors, and the distance for each node in the cluster. The cluster head will be chosen in a periodic and distributed way to consume the power in a balanced way in the IoT sensor devices inside each cluster. The comparison results confirm that our protocol can conserve power and enhance the power conservation of the network better than other approaches.
\end{abstract}

Keywords: Sensor Networks, DBSCAN clustering, performance evaluation, power conservation, network lifetime, cluster head election.

\section{Introduction}

WSNs have recently gained significant attention for their implications found in various fields including ecosystem monitoring, health care, environment assessment, urban areas applications, control maintenance, and target tracking [1]. The connection of all things that the internet can monitor or control could be defined as Internet of Things (IoT), which is most preferably achieved through a wireless medium [2, 22] The network of Wireless sensors could be depicted as the set of huge sensor nodes used over a wide area for sensing and accumulating different 
data from the systems and environment, so as to be applied in a variety of uses like weather monitors, animal tracking, disaster managing, and bio-medical applications, within IoT [5, 23]. Wireless sensors could be of use with IoT applications for gathering and processing data with the extraction of valuable information to be communicated to the end user, as it can occasionally be unreachable by individuals. Therefore, WSNs are among the integrated parts of IoT applications [4]. A direct communication is made by each node over the BS as the data is distributed [5]. Through the continuity of data transmitted, the furthest node would be more likely to die earlier than others through its energy loss [9]. Consequently, the clustering process applied tends to collect nodes, forming a set of clusters for solving the problem [2]. Its main performance is remarkably improved through clustering several nodes [6]. In addition, the network keeps the demands of central organization to a minimum, inspiring the local decision-making so as to enhance the scalability. The clustering procedure tends to collect data through the active network. As well, a suitable cluster head needs to be selected for every data retrieving clusters [7], through sensor nodes to be passed to the BS [8]. The SN eventually creates clusters for the monitoring procedure and the constitution of both cluster member (CM) and Cluster Head ( $\mathrm{CH})$ [9]. The SNs contain battery sources for their performance, which makes them a tool of power starving. The main elements that influences the energy dissipating of WNS are the distance with sink, remaining energy of the node, and intra-cluster distance [10]. The remained power, distance among the core nodes in the cluster, and the members number of each core node are the three factors that the $\mathrm{CH}$ choosing is based on in our work. Thus, at the same time the elected $\mathrm{CH}$ must have a highest remained power, maximum members and finally a lowest distance to all core nodes. The power can be preserved to extend the life of the sensor device if these factors considering and the simulations show the good results in the later of this paper.

The contributions in this paper can be presented as follow.

$\rightarrow$ Distributed clustering based DBSCAN protocol is proposed for increasing the lifespan of wireless sensors of IoT Networks. This protocol is distributed on every IoT sensor device and the sensor devices are cooperated based the DBSCAN algorithm to form several groups in the network area of interest.

$\rightarrow$ Efficient periodic $\mathrm{CH}$ approach is suggested based on several criteria like remaining power, neighbors' number, and the distance for each node in the group. The cluster head will be elected periodically so as to the consumed power is balanced in the wireless sensors inside each group.

? The proposed protocol is evaluated and compared to two existing methods such as I-LEACH [10] and LEACH that presented in [14] in light of several metrics like the resting energy, network lifetime and $\mathrm{CH}$ count, etc. The comparison of simulation results illustrates that our protocol can preserve energy and increase the network lifetime better than other approaches. 
The remaining of the article is structured as follows. The related literature is presented in Section 2. The DBSCAN traditional algorithm is explained in more detail in Section 3. Section 4 introduces the proposed Energy-efficient distributed clustering based DBSCAN protocol for conserving power of wireless sensors of IoT Networks. The Results and discussion are explained in Section 5. Section 6 presents the conclusion and the planned work for the future.

\section{Related Work}

The cluster head $(\mathrm{CH})$ has been selected in a random manner, resulting in a similar likeliness for both nodes with high or low energy to become a $\mathrm{CH}$. Whenever a low energy node is elected to be a $\mathrm{CH}$, it is most likely to die soon, with its eventual effect on how robust the network is. In addition, every round varies in how many CHs it has, as well as their location [12]. As with WSNs, the main focus lies on two essential factors: reducing the consumed power, as well as extending the network lifespan. Taking LEACH protocol to be the basic algorithm, several alterations are made in light of differing applications. LEACH and its related researches have been presented [13], taking into account a number of significant parameters including the clustering method, data aggregating, scalability and mobility type. This protocol makes a random selection of $\mathrm{CHs}$ without the BS knowing any details on the network's remaining energy [11]. Therefore, the LEACH-C protocol has been suggested for addressing this issue [14].

The PSO-ECHSs are dealt with as the PSO based CHs are selected with the use of factors such as distance among nodes, remaining energy, and distance to BS [15]. An alternative optimizing method known as Grouped Grey Wolf Search Optimization has been applied in [16] selecting security-aware $\mathrm{CH}$, with the purpose of improving the network lifespan choice. The researchers proposed an alternative algorithm which first calculates the ideal cluster number, taking into account its location adaptability and data aggregating rate. Next, a new parameter is presented in light of the residual, initial and average energy consumption, as well as the node degree for selecting the $\mathrm{CH}$. A third aspect is the proposal of an unevenly self-adaptive clustering algorithm which considers the node degree in solving the "hot spot" problem. At last, a solution is suggested for the "isolated nodes problem" [17].

Jan et al. [18] present a new method known as a mutual authentication approach based on payload, as it consists of two steps selecting nodes optimality which act as $\mathrm{CH}$, being allowed to have communication with its neighbors, and the authentication of every $\mathrm{CH}$ of its near nodes for forming clusters. After the former step, a method of authenticating takes place which depends on tokens. The tokens are used in the correlation between the $\mathrm{CH}$ and the acknowledge messages it corresponds with. Authenticating the payload cluster contributes to forming clusters from close member nodes and the $\mathrm{CH}$. A comparison between the scheme of each the LEACH-C and Sec LEACH is suggested. The suggested model shows a lack in the use of an encryption method, as well as the improvement of its performance and 
comparing it to modern clustering models with a random distribution. Purohit and Bhargava introduced the Multi hop routing scheme [19] where the one hop transmission is transformed into multi hop way, with the purpose of reducing the consumed energy by a sensor. This helps in obtaining the effective use of energy. The experiment resulted in the improvement of performance regarding the time for the first node to die (FND). This had a clear influence on improving the network energy effectively. The main aim of this proposed idea is inter-cluster communicating, but still the time needed to receive messages is rather higher, negatively affecting its work. The main use of WSNs is receiving information for doing several performances, mainly in light of the data received. The decrease of messages count displays the inactive nodes in an automatic way, eventually declining the network's general performance. The work in [10] is introduced an improved method of the LEACH named I-LEACH. It is limited the selection of the cluster head using a certain threshold with concurrently changing the level of power between the nodes. The results explain better performance with the original protocol.

SHORTCOMINGS. Despite many clustering approaches were proposed for grouping the sensor nodes in the WSNs, but none of them can ensure an optimal energy-saving and this would result in a shorter lifespan of the WSN.

OUR APPROACH. This paper suggested a distributed DBSCAN protocol for preserving the power of sensor devices in IoT Networks. This protocol is executed at each IoT wireless sensor and the devices apply the DBSCAN scheme to divide the network into groups in a distributed way. An efficient cluster head strategy is proposed based on certain criteria like remaining energy, number of neighbors, and the distance for each node in the cluster. The cluster head will be selected in a periodic and distributed way to conserve the power in a balanced way in the IoT wireless sensors inside each cluster.

\section{The DBSCAN Algorithm}

The DBSCAN approach identifies clusters within huge spatial datasets by taking into account the local density of its elements, with the use of a single input parameter. In addition, a suitable parameter value is suggested for the user, so little knowledge about the domain itself is needed. The aim of DBSCAN is categorizing them into clusters apart, eventually defining the differing classifications [20].

The next section suggests a description of the manner in which the DBSCAN algorithm performs. In traditional DBSCAN, the user requires two parameters to be defined: the neighborhood range e and MinPts that refers to the lower required number of points to construct a new cluster. In the beginning, the scheme chooses one-point P randomly and then calculate the distance among this selected point and the rest point in the dataset. The neighborhood condition between the point $\mathrm{P}$ and any other point in the dataset if the distance between them is less or equal to e. If the number of points which are in the neighborhood range of point $\mathrm{P}$ greater than or equal to MinPts, the new cluster will be constructed, otherwise, these points as noise 
are labeled. This mean that noise points can later be within other clusters if they satisfy the condition of the required MinPts in the neighborhood range of the newly selected point. After that, the DBSCAN scheme will check if it is possible to extend this cluster or it chooses another point from the outside of the current cluster. The checking is done by verifying both minPts and distance conditions if they are satisfied for each point in range of the cluster. If these conditions are satisfied, DBSCAN scheme extend this cluster to each point in the neighborhood range of point $\mathrm{P}$. The extension of the cluster will be stopped and each point will be labeled as a visited point if the cluster expanded to the required MinPts. The DBSCAN scheme then chooses another random not visited point from the dataset and repeats the same above scheme. The DBSCAN scheme will be stopped if there is no point labeled as not visited. Algorithm 1 illustrates the DBSCAN scheme with its expanding function. The time requirement of the DBSCAN scheme is O(n2), where $\mathrm{n}$ refers to the size of the dataset. The time complexity will be decreased to the $\mathrm{O}(\mathrm{n} \log n)$ if the spatial indexing is utilized [21].

\section{Energy-Efficient Distributed DBSCAN Protocol}

This research aims at proposing a distributed DBSCAN protocol for maximizing the wireless sensors lifetime. This protocol is distributed at every sensor device deployed in the monitored area. The proposed protocol involving two steps: setup and steady-state. For the sake of simplicity, the proposed distributed DBSCAN protocol is named as DBSCAN protocol in this paper. The DBSCAN protocol is presented in Figure (1).

In the Setup phase, When the sensor devices are deployed in the working area, it is supposed that every sensor device knows its location. According to Dbscan algorithm, each sensor implements the algorithm of DbScan as follow:

- Each sensor node will perform the same test whether it is Core Point or not, according to the principle of the Dbscan algorithm, as it scans the surrounding area to find out the number of sensors that are within the sensing range and that must be greater or equal to a specific parameter.

- As for the sensor nodes that are within the sensing range for Core Point, it will be its member.

- If this core point does not belong to any cluster, it forms a new cluster, otherwise it remains with the same cluster.

- The core point sends a message to all its members to be included in the same its cluster.

- Repeat steps 1-5 until all sensor nodes are passed. 


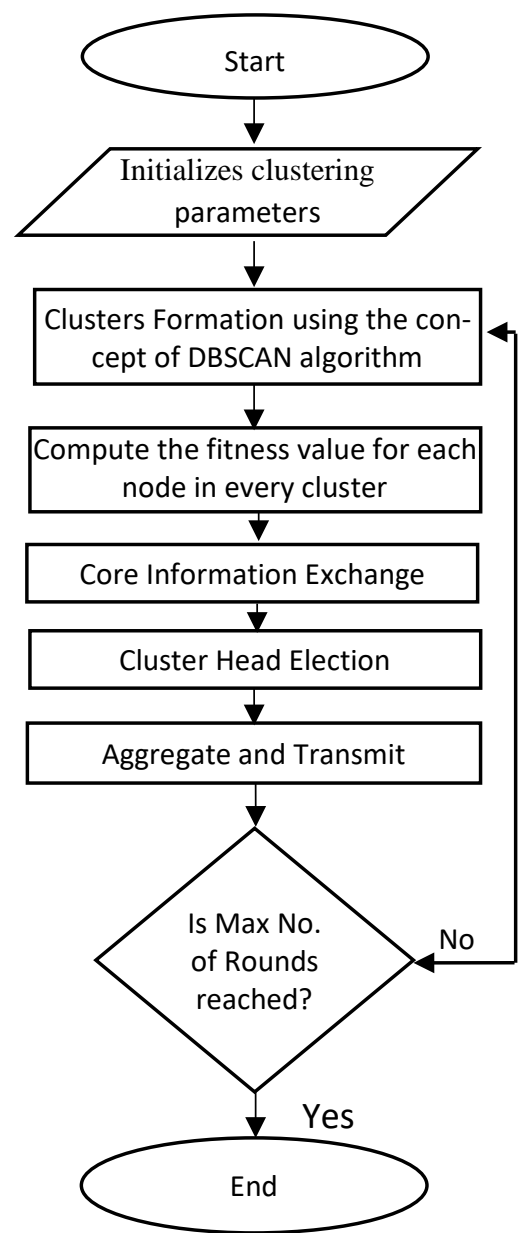

Fig. 1. DBSCAN Protocol

Algorithm 1 explains the Distributed DBSCAN algorithm that will be executed in every sensor node sj.

Algorithm 1. Distributed DBSCAN (sj)

Input: N: number of neighbor nodes, Sr: sensing range, minNodes: minimum number of nodes to create cluster.

Output: sj.rejon: the cluster number for node sj.

1: $\quad$ while $R E j \geq$ Ethr do

2: If sj Receive MemberPacket from si then

3: $\quad$ Mark sj as member to the Core si ;

4: $\quad$ Update REj;

5: $\quad$ end 


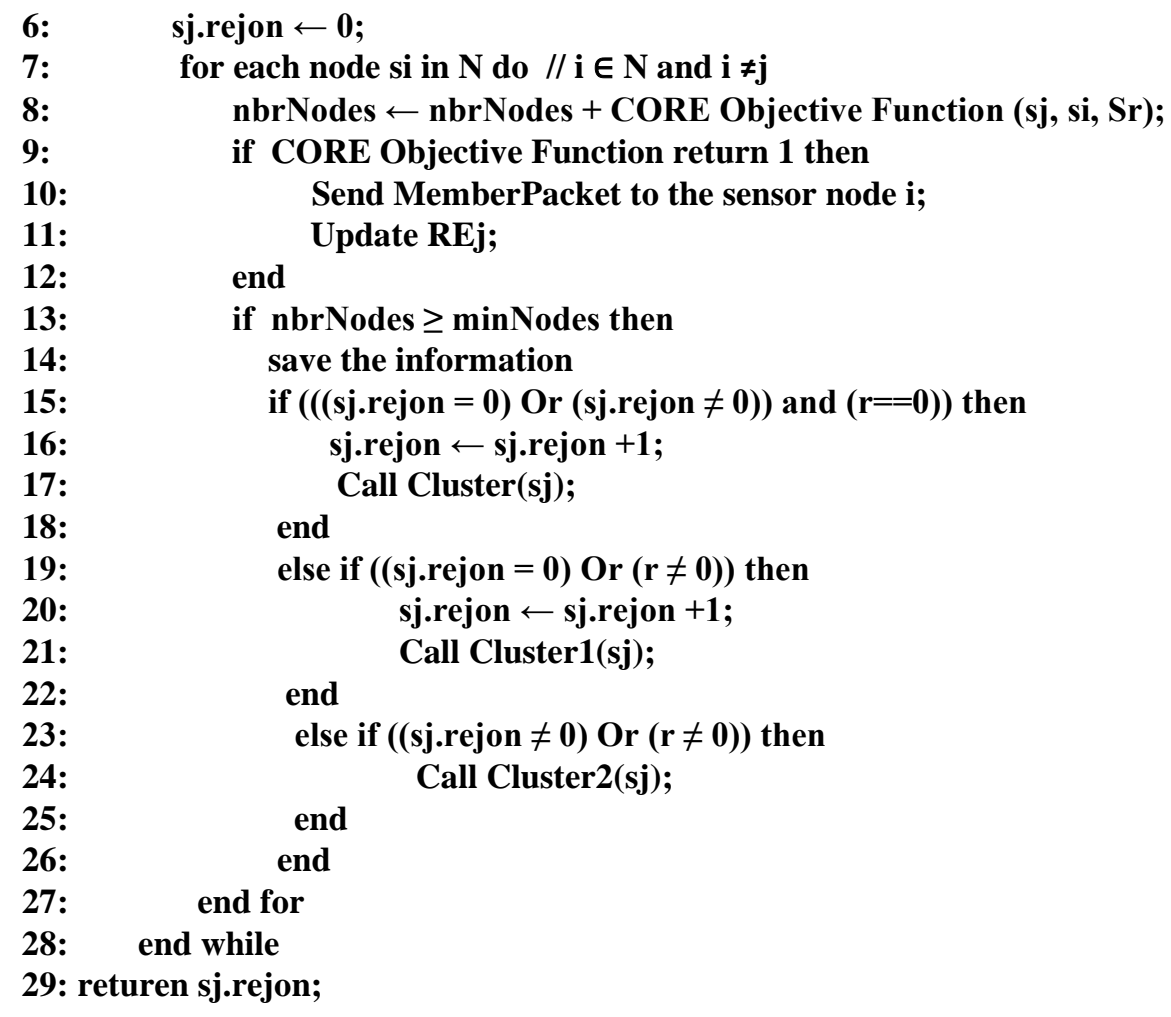

CORE Objective Function return 1 and $r=0$ if the sensor node $i$ is within the sensing range $\mathrm{Sr}$ and it is not a member in other clusters. Otherwise, CORE Objective Function returns 0 and $r=1$. The function Cluster put any neighbor node within the sensing range of $\mathrm{sj}$ in the same cluster and sj send MemberPacket to the sensor node i to inform it that it becomes a member in the same cluster of sj. The function Cluster1 put any neighbor node within the sensing range of $\mathrm{sj}$ and it has not assigned to any cluster in the same cluster of sensor node $\mathrm{j}$. The function Cluster2 put any neighbor node within the sensing range of sj and it has not assigned to any cluster (or it is assigned to the cluster of sensor node $\mathrm{j}$ ) in the same cluster of sensor node j. After achieving the functions Cluster, Cluster1, and Cluster2, the remaining energy of the sensor node $\mathrm{j}$ will be updated due to sending $\mathrm{a}$ MemberPacket to the sensor node $\mathrm{i}$ to inform it that it becomes a member in the same cluster of sj.

After the stage of creating clusters, the exchange of information between the core points (nodes) is done inside the single cluster, where each core point sends a message to all the core points inside the Cluster, it contains all the necessary information inside like remained power, status, location, number of members, total wireless sensors number in the group (cluster), and etc. Every core sensor node 
inside each cluster will include the information of other core nodes in the same cluster, therefore, every core node in the same cluster will execute the Equation (1) for the information of each member inside the core node. The core node that give the better value of the Equation (1) will be selected as a cluster head in the current cluster for this round. All the core nodes inside the cluster will achieve the same computation and will give the same results for the winner core node. This will be implemented in distributed way and every core node will know if it is a cluster head or not.

FitVal $_{j}=\frac{E_{\text {remaining }}}{E_{\text {initial }}}+\left(1-\sum_{j \in N}\left|S_{j}(x, y)-S_{i}(x, y)\right|\right)+\frac{S_{j}(\text { Members })}{\text { Cluster }(\text { Members })}$

Where, E_remaining is the remaining power of the wireless node $\mathrm{j}, \mathrm{E}$ _initial is the initial energy value of sensor node $\mathrm{j}, \mathrm{N}$ is the number of core nodes in the current cluster, $\mathrm{S}_{-} \mathrm{j}(\mathrm{x}, \mathrm{y})$ and $\mathrm{S} \_\mathrm{i}(\mathrm{x}, \mathrm{y})$ refer to the positions of core nodes $\mathrm{S}$ _jand $\mathrm{S} \_\mathrm{i}$ respectively. $S_{\_} j$ (Members) refers to the number of nodes member of core node $j$, Cluster(Members) refers to the total number of nodes in the cluster.

In Steady phase, after clusters formation and fixing the TDMA schedule, the process of transmitting data may start. With the assumption that all nodes contain data that requires to be sent to the $\mathrm{CH}$, this sending will occur within its allocated time. The cluster head in its turn allocates a TDMA schedule to the actively participating members in order that the data transmitting process is managed and the consumed power is limited to a minimum. Based on the active/idle status. The data is transmitted during the steady-state stage with regards to the timespan assigned for every member node. The power supply of any idle node will be turned off but the $\mathrm{CH}$, which awaits the data from the member nodes. After delivering data through nodes, the $\mathrm{CH}$ initiates a data aggregating process followed by transmitting this data to the BS. The algorithm eventually returns to the setup iterating stage so as to select a different CHs group, followed by the steady stage, and so on.

\section{Performance Evaluation, Analysis, and Discussion}

This section focuses on evaluating the proposed distributed DBSCAN protocol using different performance metrics like cluster count, remaining energy, dead nodes number, packets number transmitted to cluster head, number of packets sent to cluster sink, and network lifetime. The conduction of the simulation results is performed by means of a C++ custom simulator for 2500 iterating round, so that several plots are obtained. The sensor nodes are deployed in the monitored area in a random manner. The location of the sink is in the central of the monitored area, with no limitation on energy, normal nodes would have its limitations in terms of energy, memory, and processing capabilities. The suggested protocol is applied for generating the results with regards to the parameters referred to in Table (1). 
Table 1. Stimulation Parameters

\begin{tabular}{|c|c|c|}
\hline Symbol & Describtion & Value \\
\hline $\mathrm{X} 1_{\mathrm{m}}$ & distance at X-axis & $400 \mathrm{~m}$ \\
\hline $\mathrm{Y} 1_{\mathrm{m}}$ & distance at Y-axis & $400 \mathrm{~m}$ \\
\hline $\mathrm{Ns}$ & WSN size & 100 nodes \\
& & $0.5 \mathrm{~J}$ \\
\hline $\mathrm{P}_{\mathrm{T} x}, \mathrm{P}_{\mathrm{Rx}}$ & The initial energy & \\
\hline $\mathrm{E}_{\mathrm{mp}}$ & Energy consumption for receiving & $0.0013 / \mathrm{pJ} / \mathrm{bit} / \mathrm{m} 4$ \\
\hline $\mathrm{E}_{\mathrm{fs}}$ & Energy dispersion:free space model & $10 / \mathrm{pJ} / \mathrm{bit} / \mathrm{m} 2$ \\
\hline $\mathrm{E}_{\mathrm{amp}}$ & Energy dispersion:power amplifier & $100 / \mathrm{pJ} / \mathrm{bit} / \mathrm{m} 2$ \\
& & \\
\hline $\mathrm{E}_{\mathrm{DA}}$ & Energy consumption for collection & $5 / \mathrm{nJ} / \mathrm{bit}$ \\
\hline $\mathrm{d}_{0}$ & & $87 \mathrm{~m}$ \\
\hline $\mathrm{I}$ & Reference distance & $4000 \mathrm{bits}$ \\
\hline
\end{tabular}

As for this work, the packet size tends to be relatively larger, namely be 4000 bits. The proposed protocol in this paper is named as DBSCAN. The DBSCAN protocol is applied the same energy consumption model that employed in [10]. An obvious result of stimulation is that the DBSCAN outperforms the I-LEACH [10] and LEACH [14] protocols in light of several performance metrics. The number of CHs is found to extend up to 2000 rounds for the DBSCAN, whereas it reaches only 1750 and 850 rounds for I-LEACH and LEACH respectively. Figure 2 shows the cluster count for the LEACH, I-LEACH, and DBSCAN.

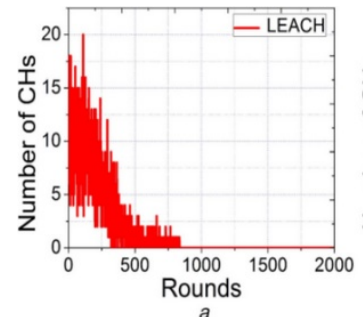

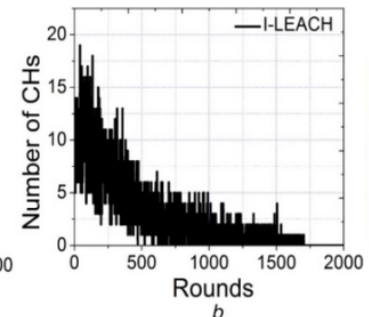

$b$

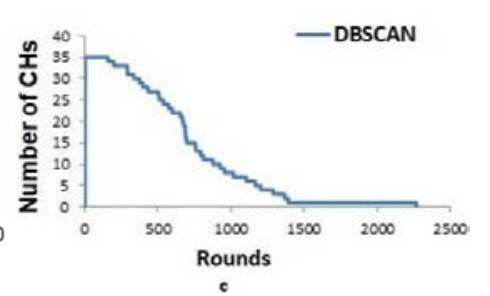

Fig. 2. CH Count (a) LEACH, (b) I-LEACH, (c) DBSCAN

Similarly, the stimulation results in Figure 3a present the fact that at just "500 rounds", the average power of LEACH reaches 0 , while I-LEACH goes on to 
"1250 rounds". The DBSCAN continues to 1750 . The amount of data packets transmitted to BS within LEACH and I-LEACH reaches maximally $(0.5 \times 104)$ and $(1.75 \times 104)$ respectively. The rise in rounds leads to the energy depletion of sensor nodes, until they die.

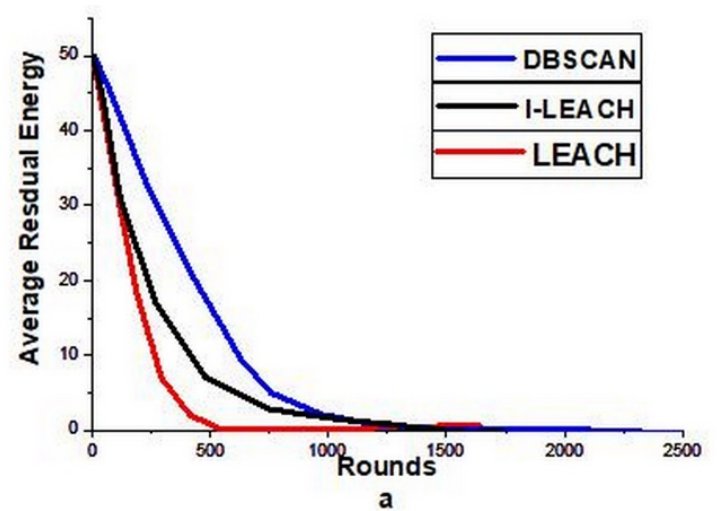

Fig. 3. Network Performance (a) Average Residual Energy

Figure 4a illustrates the network Lifetime through the representation of dead nodes. After 750 rounds, the number of nodes alive levels out at 0 for LEACH, whereas a few nodes remain active till 1500 rounds with I-LEACH. The DBSCAN, on the other hand, has several nodes that remain active till 2000 rounds.

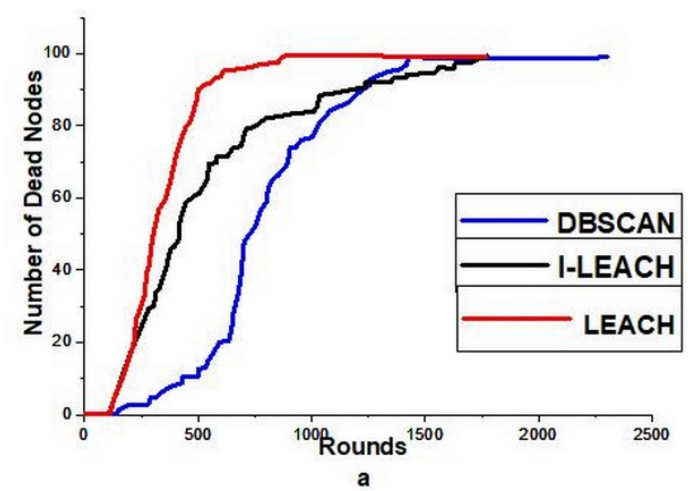

Fig. 4. Lifetime Metrics (a) Dead nodes

As for the DBSCAN, the value may reach $\left(2 \times 10^{4}\right)$, as is shown in Figure 5a. A similar increase in data packets transmitted to Cluster Head $(\mathrm{CH})$ is noticed in Figure 5b, proving the effectiveness of the suggested protocol. This illustrates the whole situation for the maximization of the network lifespan, merely due to assigning various energy levels for differing communicating modes in the network. 

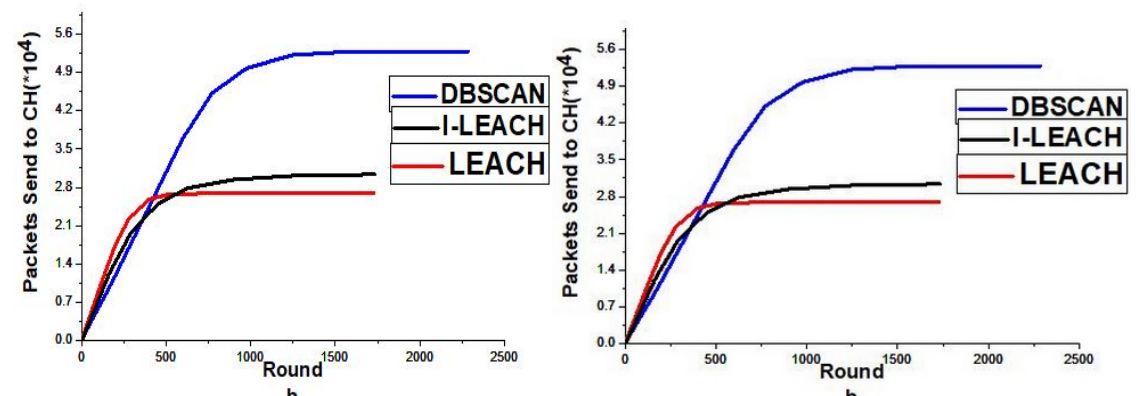

Fig. 5. Sent Packets (a) to BS, (b) to $\mathrm{CH}$

Studying the algorithm behavior in different uses requires the comparison of lifespan metrics with regards to the "first node dead (FND)" and "last node dead (LND)", as illustrated in Table (2). Simulation is performed with regards to three differing areas $(100,200$, and 400$) \mathrm{m} 2$ with a network that is poorly to richly deployed with sensors. Keeping the initiated energy at $(0.5 \mathrm{~J})$ on a poorly deployed area of $100 \mathrm{~m} 2$ improves the network timespan to 1.16, 1.36, and 1.3 for I-LEACH, LEACH, and CPCHSA respectively. There is a positive relation between the lifespan and number of nodes (keeping the area and energy constant), as it increases to 1.04, 1.87, and 1.19 times the value for I-LEACH, EECS and LEACH, respectively. Doubling the area to 100 nodes, results in a lifespan of 1.16, 1.3, and 1.47 times the value for I-LEACH, ModLeach and LEACH respectively. At 400 nodes, the lifespan is 1.23, 1.4, and 1.61 times the value for I-LEACH, ModLeach, and LEACH subsequently. With the initiated energy of $1 \mathrm{~J}$ for 1000 nodes, the lifespan is 1.4, 1.4 and 3.5 times the value for I-LEACH, LEACH and EECS. Comparing the DBSCAN to I-LEACH and LEACH over a wider area of $400 \mathrm{~m} 2$ shows the increase of the stability period by $(1.23,2.6)$ and $(1.28,1.7)$ and $(1.58$, 1.7) times for 100,400 and 1000 nodes, respectively. One can therefore draw the conclusion that the suggested protocol proves a more favorable performance with both smaller and larger areas, regardless whether the networks were poorly or richly covered with nodes.

Table 2. The Different Scenarios for the Network Lifetime

\begin{tabular}{|l|l|l|l|l|l|l|l|l|l|l|}
\hline \multirow{2}{*}{ Nodes } & \multirow{2}{*}{ Energy } & \multirow{2}{*}{ Area } & \multicolumn{2}{|c|}{ LEACH } & \multicolumn{2}{|c|}{ ILEACH } & \multicolumn{2}{|c|}{ DBSCAN } & \multicolumn{2}{l|}{$\begin{array}{l}\text { Other } \\
\text { protocols }\end{array}$} \\
\cline { 4 - 10 } & & & FDN & LDN & FDN & LDN & FD & LD & FDN & LDN \\
\hline 100 & 0.5 & 100 & 980 & 1450 & 1050 & 1700 & 1150 & 1972 & $\begin{array}{l}600 \\
{[45]}\end{array}$ & $\begin{array}{l}1500 \\
{[45]}\end{array}$ \\
\hline & & 200 & 780 & 1150 & 850 & 1450 & 676 & 1685 & $\begin{array}{l}200 \\
{[46]}\end{array}$ & $\begin{array}{l}1300 \\
{[46]}\end{array}$ \\
\hline & & 400 & 100 & 800 & 98 & 1700 & 194 & 2092 & - & - \\
\hline 400 & 0.5 & 100 & 1000 & 1500 & 1100 & 1700 & 1171 & 1778 & $\begin{array}{l}820 \\
{[47]}\end{array}$ & $\begin{array}{l}950 \\
{[47]}\end{array}$ \\
\hline & & 200 & 850 & 1300 & 900 & 1700 & 681 & 2093 & $\begin{array}{l}190 \\
{[46]}\end{array}$ & $\begin{array}{l}1500[4 \\
6]\end{array}$ \\
\hline & & 400 & 100 & 1200 & 100 & 1600 & 316 & 2049 & - & - \\
\hline
\end{tabular}




\begin{tabular}{|l|l|l|l|l|l|l|l|l|l|l|}
\hline 1000 & 1 & 100 & 2000 & 2700 & 2000 & 2700 & 2005 & 4095 & - & - \\
\hline & & 200 & 1700 & 2600 & 1700 & 2650 & 1121 & 3651 & $\begin{array}{l}810 \\
{[47]}\end{array}$ & $\begin{array}{l}1050 \\
{[47]}\end{array}$ \\
\hline & & 400 & 300 & 2500 & 300 & 2700 & 842 & 4284 & - & - \\
\hline
\end{tabular}

\section{Conclusion}

The clustering algorithms still represent a significant part in the field of WSNs and it gets a great consideration by many researchers in the WSN world. This paper suggested a distributed DBSCAN protocol for extending the lifetime of wireless sensors of IoT Networks. This protocol is distributed on every IoT sensor device and the sensor devices are cooperated based on the DBSCAN algorithm to form the clusters in the network in a distributed way. The efficient periodic cluster head strategy is introduced based on certain criteria like remaining power, a number of neighbors, and the distance for each node in the cluster. The cluster head will be elected periodically and in a distributed way so as to the consumed power is balanced in the sensors inside each cluster. The proposed protocol is evaluated and compared to two existing methods using several performance metrics like the resting energy, network lifetime and $\mathrm{CH}$ count, etc. The comparison results show that the suggested protocol can preserve power and improve the network lifetime better than other approaches.

\section{References}

1. R. Alhussaini, A. K. Idrees, and M. A. Salman, "Data Transmission Protocol for Reducing the Energy Consumption in Wireless Sensor Networks,” New Trends in Information and Communications Technology Applications, pp. 35-49, 2018.

2. A. K. Idrees, K. Deschinkel, M. Salomon, and R. Couturier, "Multiround Distributed Lifetime Coverage Optimization protocol in wireless sensor networks," The Journal of Supercomputing, vol. 74, no. 5, pp. 1949-1972, Apr. 2017.

3. A. K. Idrees, W. L. Al-Yaseen, M. A. Taam, and O. Zahwe, "Distributed Data Aggregation based Modified K-means technique for energy conservation in periodic wireless sensor networks,” 2018 IEEE Middle East and North Africa Communications Conference (MENACOMM), 2018.

4. H. Harb, A. K. Idrees, A. Jaber, A. Makhoul, O. Zahwe, and M. A. Taam, "Wireless Sensor Networks: A Big Data Source in Internet of Things,” International Journal of Sensors, Wireless Communications and Control, vol. 7, no. 2, 2018.

5. A. K. Idrees, H. Harb, A. Jaber, O. Zahwe, and M. A. Taam, "Adaptive distributed energy-saving data gathering technique for wireless sensor networks," 2017 IEEE 13th International Conference on Wireless and Mobile Computing, Networking and Communications (WiMob), 2017.

6. H. Fotouhi, M. Alves, M. Z. Zamalloa, and A. Koubaa, "Reliable and Fast Hand-Offs in Low-Power Wireless Networks," IEEE Transactions on Mobile Computing, vol. 13, no. 11, pp. 2620-2633, 2014.

7. N. M. Ranjan and R. S. Prasad, "LFNN: Lion fuzzy neural network-based evolutionary 
model for text classification using context and sense based features,” Applied Soft Computing, vol. 71, pp. 994-1008, 2018.

8. D. Geeta, N. Nalini, and R. C. Biradar, "Fault tolerance in wireless sensor network using hand-off and dynamic power adjustment approach,” Journal of Network and Computer Applications, vol. 36, no. 4, pp. 1174-1185, 2013.

9. A. Chamam, S. Pierre. "A distributed energy-efficient clustering protocol for wireless sensor networks".Computers \& Electrical Engineering, 36(2), 303-312, 2016

10. T. M. Behera, U. C. Samal, and S. K. Mohapatra, "Energy-efficient modified LEACH protocol for IoT application,” IET Wireless Sensor Systems, vol. 8, no. 5, pp. 223-228, Jan. 2018.

11. W. R. Heinzelman, A. Chandrakasan, H. Balakrishnan. 'Energy-efficient communication protocol for wireless microsensor networks'. Proc. of the 33rd Annual Hawaii Int. Conf. on System Sciences, Maui, HI, USA, vol. 2, p. 10, 2000.

12. S. K. Singh, P. Kumar, and J. P. Singh, "A Survey on Successors of LEACH Protocol," IEEE Access, vol. 5, pp. 4298-4328, 2017.

13. R. P. Mahapatra and R. K. Yadav, "Descendant of LEACH Based Routing Protocols in Wireless Sensor Networks,” Procedia Computer Science, vol. 57, pp. 1005-1014, 2015

14. W. Heinzelman, A. Chandrakasan, and H. Balakrishnan, "An application-specific protocol architecture for wireless microsensor networks," IEEE Transactions on Wireless Communications, vol. 1, no. 4, pp. 660-670, 2002.

15. P. C. S. Rao, P. K. Jana, and H. Banka, "A particle swarm optimization based energy efficient cluster head selection algorithm for wireless sensor networks," Wireless Networks, vol. 23, no. 7, pp. 2005-2020, 2016.

16. A. Shankar, N. Jaisankar, M. S. Khan, R. Patan, and B. Balamurugan, "Hybrid model for security-aware cluster head selection in wireless sensor networks," IET Wireless Sensor Systems, vol. 9, no. 2, pp. 68-76, Jan. 2019.

17. J. Li, X. Jiang, and I.-T. Lu, "Energy Balance Routing Algorithm Based on Virtual MIMO Scheme for Wireless Sensor Networks,” Journal of Sensors, vol. 2014, pp. 1-7, 2014.

18. M. A. Jan, P. Nanda, M. Usman, X. He. "PAWN: a payload-based mutual authentication scheme for wireless sensor networks". Concurrency and Computation: Practice and Experience, 29(17), 2017.

19. R. Purohit and D. Bhargava, "An illustration to secured way of data mining using privacy preserving data mining," Journal of Statistics and Management Systems, vol. 20, no. 4, pp. 637-645, Apr. 2017.

20. M. Ester, H. P. Kriegel, J. Sander, X. Xu. "A density-based algorithm for discovering clusters in large spatial Databases with Noise". In Proc. 1996 Int. Conf. Knowledg Discovery and Data Mining (KDD ’96) (pp. 226-231), 1996.

21. D. Han, A. Agrawal, W.-K. Liao, and A. Choudhary, "A Novel Scalable DBSCAN Algorithm with Spark,” 2016 IEEE International Parallel and Distributed Processing Symposium Workshops (IPDPSW), 2016.

22. Raj, Jennifer S. "QoS optimization of energy efficient routing in IoT wireless sensor networks." Journal of ISMAC 1, no. 01 (2019): 12-23.

23. Duraipandian, M., and Mr R. Vinothkanna. "Cloud based Internet of Things for smart connected objects." Journal of ISMAC 1, no. 02 (2019): 111-119. 